\title{
Does breast-feeding reduce offspring junk food consumption during childhood? Examinations by socio-economic status and race/ethnicity
}

\author{
Dylan B Jackson ${ }^{1, *}$ and Kecia R Johnson ${ }^{2}$ \\ 'Department of Criminal Justice, The University of Texas at San Antonio, 501 W. Cesar E Chavez Blvd, San Antonio, \\ TX 78207, USA: ${ }^{2}$ Department of Sociology, Mississippi State University, Mississippi State, MS, USA
}

Submitted 19 August 2016: Final revision received 7 November 2016: Accepted 30 November 2016: First published online 20 January 2017

\begin{abstract}
Objective: To examine whether breast-feeding duration and socio-economic status (SES) interact to predict junk food consumption among offspring and whether the interaction differs across racial/ethnic groups.

Design: Survey research using a longitudinal panel design. Hierarchical linear regression was used to analyse the data.

Setting: In-home interviews with the child's parents over a 5-year period across the USA.

Subjects: Approximately 10000 American children from the Early Childhood Longitudinal Study: Birth Cohort (ECLS-B).

Results: The findings revealed that longer breast-feeding durations correspond to lower levels of junk food consumption, but that this relationship emerges consistently only among low-SES blacks.

Conclusions: Efforts to promote breast-feeding among low-SES black women may have the added benefit of reducing their children's junk food intake, and may thereby promote their general health and well-being. Future research should seek to explore the mechanisms by which breast-feeding might benefit the dietary habits of low-SES black children.
\end{abstract}

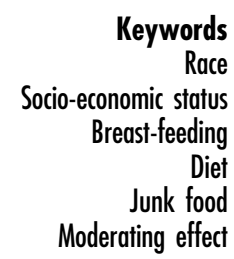

Breast-feeding has been associated with a host of child health benefits, including improved immunity ${ }^{(1)}$, diminished risk of infection ${ }^{(2)}$, lower blood pressure ${ }^{(3)}$, reduced risk of respiratory illness ${ }^{(4)}$ and decreased likelihood of childhood overweight/obesity ${ }^{(5,6)}$. Despite the long line of literature linking breast-feeding to various health outcomes in offspring, only recently have scholars considered whether offspring dietary patterns during childhood are also predicted by breast-feeding history ${ }^{(7-12)}$. This tendency to overlook diet is somewhat surprising, as research has found both breast-feeding ${ }^{(5,6)}$ and dietary habits ${ }^{(13,14)}$ to be associated with overweight/obesity. Even so, this small but growing body of research is quite consistent in its findings, suggesting that breast-feeding does indeed improve infant and child dietary patterns ${ }^{(7-12)}$. This association appears to hold across multiple age groups and dietary components and may be partly explained by (i) lower maternal feeding restriction and enhanced infant self-regulation of intake ${ }^{(15)}$ and/or (ii) repeated exposure of the breast-fed infant to the array of flavours found in the mother's diet that are transmitted through breast milk ${ }^{(11)}$.

Apart from the studies linking breast-feeding to healthrelated outcomes, scholars have also detected associations between breast-feeding and specific demographic variables, namely race/ethnicity ${ }^{(16,17)}$ and socio-economic status (SES) ${ }^{(18-20)}$. Black women have historically been, and currently are, least likely to initiate and continue breast-feeding compared with other racial and ethnic groups in the USA ${ }^{(16,17)}$. Possible reasons for racial/ethnic disparities in breast-feeding include, but are not limited to, differential preferences for bottle-feeding, the availability of free formula from the Special Supplemental Nutrition Program for Women, Infants and Children (WIC), variation in comfort levels with breast-feeding $v$. formula-feeding, limited availability of WIC breast-feeding support for minority women, degree of family support and differences in breast-feeding ambivalence ${ }^{(16,21,22)}$. Relatedly, women of lower SES are also less likely to breast-feed their infants in the $\mathrm{USA}^{(18-20)}$, whereas high levels of educational attainment and work in executive occupations among women and/or their partners increase the likelihood of breast-feeding ${ }^{(16,20)}$. Highly educated mothers may be (i) more likely to search out information on the health aspects of infant feeding choices and (ii) better able to afford feeding supplies ${ }^{(18,20,23)}$. Women working in lowerstatus occupations, however, may find expressing breast 
milk while at work more challenging, especially when their occupations are hazardous ${ }^{(20,24)}$.

Whatever the reasons for the disparities in breastfeeding, race/ethnicity and SES may be worth considering when examining associations between breast-feeding and child health outcomes, particularly since such outcomes, including obesity and diet, also vary by race/ethnicity and $\mathrm{SES}^{(25-27)}$. A handful of scholars have employed this line of reasoning by examining whether the influence of breast-feeding on overweight/obesity varies as a function of race/ethnicity and/or SES ${ }^{(28-32)}$. The results, however, have not been entirely consistent. One study found no evidence that the influence of breast-feeding on childhood obesity varies by level of SES ${ }^{(28)}$. However, in the case of race/ethnicity, some scholars have found a significant association between breast-feeding and overweight/ obesity for whites and blacks, but not Hispanics ${ }^{(31)}$. Still, the findings are far from uniform, with one group of researchers detecting an association between breastfeeding and overweight/obesity among non-Hispanic whites $^{(32)}$, and another among Hispanics only ${ }^{(29)}$. It therefore remains unclear whether race/ethnicity moderates the link between breast-feeding and child health, at least in the case of overweight/obesity.

Despite a number of studies examining race/ethnicity and SES as moderators of the link between breast-feeding and overweight/obesity, researchers have yet to consider whether the association between child diet and breastfeeding might differ across subsets of the population distinguished by SES and race/ethnicity. Historically, breast-feeding has varied across these racial/ethnic and socio-economic groups, and, in some studies, the benefits of breast-feeding for overweight/obesity has also varied across these groups. The current study aimed to extend this body of literature by examining whether junk food consumption during childhood varies as a function of breast-feeding history and whether the pattern of results differs on the basis of specific demographic features known to be associated with both breast-feeding practices and childhood dietary patterns (i.e. SES and race/ ethnicity).

\section{Materials and methods}

\section{Participants}

The present study employed data from the Early Childhood Longitudinal Study: Birth Cohort (ECLS-B), a nationally representative study of children born in the USA in 2001. The ECLS-B follows a large cohort of children from birth through their kindergarten school year (2007/ 2008). The study sampled birth certificates registered with the National Center for Health Statistics using the technique of stratified sampling, which permitted the inclusion of approximately 99\% of US births during 2001 in the sampling frame. Children were deemed ineligible if they met any of the following criteria: (i) they died before the age of 9 months; (ii) they were adopted before the age of 9 months; or (iii) their mothers were younger than 15 years at the time of birth. Birth certificates, direct child assessment, parent interviews and teacher questionnaires were all employed to obtain the data. In total, five waves of data have been collected. Importantly, the data contain various details concerning both the health and nutrition of the child, including her/his duration of breast-feeding and junk food intake during childhood. Various familial, developmental and demographic details are also included. These features of the data make the ECLS-B well suited to the present study.

\section{Measures}

\section{Junk food consumption}

At the fourth and fifth waves of data collection, when the focal children were in kindergarten, parents were asked to report how frequently their child ingested a number of junk foods during the $7 \mathrm{~d}$ prior to the interview. The questions covered various domains of junk food consumption, including fast-food consumption, soda/ sugary beverage consumption, salty snack consumption and sweets consumption. Specifically, parents were asked the following questions.

1. Fast-food consumption: 'During the past 7 days, how many times did your child eat a meal or snack from a fast food restaurant with no wait service such as McDonald's, Pizza Hut, Burger King, Kentucky Fried Chicken, Taco Bell, Wendy's and so on? Consider both eating out, carry out, and delivery of meals in your response.'

2. Soda consumption: 'During the past 7 days, how many times did your child drink Soda pop (for example, Coke, Pepsi, or Mountain Dew), sports drinks (for example, Gatorade) or fruit drinks that are not $100 \%$ fruit juice (for example, Kool-Aid, Sunny Delight, Hi-C, Fruitopia, or Fruitworks)?'

3. Salty snack consumption: 'During the past 7 days, how many times did your child eat potato chips, corn chips such as Fritos or Doritos, Cheetos, pretzels, popcorn, crackers or other salty snack foods?'

4. Sweets consumption: 'During the past 7 days, how many times did your child eat candy (including Fruit Roll-Ups and similar items), ice cream, cookies, cakes, brownies, or other sweets?'

Response options to each of these items included 'not at all in the past 7 days' ( 7 ), ' $1-3$ times during the past 7 days' (5), ' 4 to 6 times during the past 7 days' (6), ' 1 time per day' (1), '2 times per day' (2), '3 times per day' (3) and ' 4 or more times per day' (4). The items were ultimately recoded so that higher scores were assigned to children whose parents reported that they ate junk foods more frequently, with 'not at all in the past 7 days' being 
assigned the lowest value ( 0 ) and ' 4 or more times per day' being assigned the highest value (6). Consequently, possible scores on each item ranged from 0 to 6 . Junk food items were then summed and averaged to create a composite measure of junk food consumption across junk food domains. Importantly, children who had not entered kindergarten by wave 4 were not included in the fourth wave of data collection, but were instead included at wave 5 (i.e. the wave during which they entered kindergarten). Thus, junk food consumption was measured at wave 4 for most children and at wave 5 for a smaller portion of children, contingent on their timing of entry into kindergarten.*

\section{Breast-feeding duration}

At waves 1 and 2 of data collection, when children were approximately 9 months and 2 years of age, mothers were asked three questions about breast-feeding their child. The first question was: 'Did you ever breast-feed (the child)? Yes (1) or No (2)'. The follow-up question was: 'Are you still breast-feeding (the child) now? Yes (1) or No (2)'. Mothers who responded that they had terminated breastfeeding were then asked: 'For how many months did you breast-feed (the child)?', to which mothers responded with the number of months the child was breast-fed. Data from waves 1 and 2 were used to construct a variable that measured the duration of breast-feeding (in months) for each child, ranging from 0 to 21 . Children in the final sample who were still breast-feeding at wave 2 were all at least 21 months old. As a result, focal children whose mothers reported that they were still breast-feeding at wave 2 were assigned a value of 21 . However, it is important to note that being assigned a value of 21 on this variable indicates 21 months or more of breast-feeding, as the data provide no means of determining the precise duration of breast-feeding for children who were still breast-feeding at wave 2 . Additionally, children who were no longer breast-feeding at wave 2 , but had discontinued breast-feeding at the age of 21 months or more, were also assigned a value of 21 on this variable. Importantly, just under $3 \%$ of the full sample was reported as being breastfed for 21 months or more. $\dagger$ Despite this slight truncation of the measure, our continuous, dose-response measure of breast-feeding is an improvement over most measures used in prior studies, at least in terms of specificity of duration $^{(33-36)}$.

\section{Socio-economic status}

The first key moderator of interest in the present study is SES. The ECLS-B data contain a comprehensive measure of household SES that has been utilized in prior research ${ }^{(37,38)}$. The item was constructed by ECLS-B

\footnotetext{
* Timing of entry into kindergarten did not alter the results of the study in any substantive way.

$\dagger$ Among black subjects, this number dropped to $1.2 \%$
}

researchers and is derived from the primary parent interview (i.e. usually the mother). The item includes a composite of scores pertaining to household income, maternal education, paternal (or resident partner) education and occupational prestige. In cases where the father was a non-resident, additional indicators tapping other economic resources were included in the composite measure, including monthly child support paid and other financial help from the father (e.g. childcare expenses). Ultimately, higher scores reflect a higher level of household SES. In the current study, the item was standardized for the purpose of creating multiplicative interaction terms ${ }^{(39)}$.

\section{Race/ethnicity}

The second key moderator in the present analysis is race/ ethnicity. For the present study purposes, the construct is divided into three dichotomous variables: white (nonHispanic), Hispanic and black. Importantly, race/ethnicity pertains to the mother in the current study, although in the vast majority of incidences the mother and child were concordant on race/ethnicity.

\section{Child sex}

The biological sex of the child was included as a covariate in the analysis, with male offspring being assigned a value of 1 and female offspring being assigned a value of 0 .

\section{Child age}

The child's age at wave 1 (in months) was also included as a covariate in the analysis.

\section{Child attachment security}

Considering the link between breast-feeding and attachment security ${ }^{(40)}$ and attachment security and offspring $\operatorname{diet}^{(41)}$, we opted to include a dichotomous measure of attachment security in the present analysis that was derived from the Toddler Attachment Sort-45 (TAS-45). Securely attached children were assigned a value of 1 , whereas insecurely attached children were assigned a value of 0 .

\section{Maternal age at birth}

The mother's age at birth (in years) was also included as a covariate in the analysis due to its correlation with breastfeeding practices ${ }^{(42)}$ and its potential relevance for child dietary patterns ${ }^{(43)}$.

\section{Marital status}

Marital status was also included as a covariate in the analysis, with married maternal respondents being coded as 1 and unmarried respondents being coded as 0 .

\section{Employment}

An item pertaining to the mother's employment at the first wave of data collection was also included in the analysis, 
due to the negative correlation between employment and breast-feeding duration ${ }^{(44)}$. Mothers who reported that they were employed at the first wave of data collection were assigned a value of 1 , whereas mothers who were not employed at the first wave of data collection were assigned a value of 0 .*

Participation in the Special Supplemental Nutrition Program for Women, Infants and Children

A dichotomous item tapping WIC participation during pregnancy and following childbirth was also included in the analysis, as WIC participation has historically been negatively associated with breast-feeding duration and initiation ${ }^{(45)}$. Mothers who reported that they had participated in WIC during this time were assigned a value of 1 , whereas mothers who did not participate in WIC were assigned a value of 0 .

\section{Postpartum depression}

At wave 1, parents were asked twelve questions about the extent to which they felt depressed. For instance, they were asked how frequently they felt restless, lonely, sad, depressed, unfocused and unmotivated during the week prior to the survey. Response options ranged from 'less than a day' (1) to 'most or all days' (4). Ultimately, in order to tap parents' overall depressive symptomatology, a scale was created by summing the twelve items together $(\alpha=0 \cdot 87)$.

\section{Maternal involvement}

Mothers were also asked several questions about their involvement with their infant at the first wave of data collection. To illustrate, mothers were asked how often they read books, tell stories and sing songs to their infant. Response options ranged from 1 ('not at all') to 4 ('every day'). Higher scores on this item correspond to higher levels of maternal involvement $(\alpha=0 \cdot 66)$.

\section{Food rules}

At the third wave of data collection, when focal children were approximately 4 years old, mothers were asked, 'In your house, are there rules and routines about what kinds of food the child eats?' Mothers who responded in the affirmative were assigned a value of 1 . Mothers who reported that there were no household rules concerning food were assigned a value of 0 .

\section{Family meals}

At wave 3, respondents stated the following during the interview with the mother: 'In a typical week, please tell me the number of days at least some of the family eats the evening meal together.' Response options ranged from 'one day per week' (1) to 'seven days per week' (7).

* Distinguishing between part-time and full-time work had no bearing on the results of the present study.
Thus, the item is coded so that higher scores reflect a greater number of family dinners in a typical week.

\section{Statistical analysis}

The current analysis proceeded in a series of steps. First, descriptive statistics of the all of the variables included in the analyses were calculated and examined. Next, hierarchical regression techniques (ordinary least squares) were employed to first explore whether breast-feeding duration independently predicted junk food consumption among offspring during childhood. Subsequently, the possibility of a statistical interaction between breastfeeding duration and SES in the prediction of junk food consumption was tested. Next, we divided the sample by race/ethnicity to examine (i) whether the influence of breast-feeding and SES on offspring junk food consumptions varied by race/ethnicity and (ii) whether race/ethnicity moderated any interaction between breast-feeding duration and SES. Finally, the specific domains of junk food consumption (i.e. fast foods, soda, salty snacks and sweets) were employed as outcome variables to examine whether any three-way interactions detected between breast-feeding duration, SES and race/ethnicity were robust to the type of junk food examined. In line with a large body of prior research, variables that comprise interaction terms were standardized before multiplication $^{(39)}$ and the statistical significance of relevant coefficients and interaction terms across models distinguished by race/ethnicity was determined by conducting $z$ tests for the equality of regression coefficients ${ }^{(46)}$.

\section{Results}

We proceeded with the analysis by first examining the descriptive statistics presented in Table 1 , which revealed that the average breast-feeding duration in the full sample was approximately 4 months. Roughly $45 \%$ of mothers were white, whereas 17 and $16 \%$ were Hispanic and black, respectively. Children, on average, ate sweets most frequently ( mean $=2 \cdot 22$ ) and fast foods least frequently (mean $=0.91$ ). Male children made up $51 \%$ of focal subjects and the approximate age of children at wave $4 / 5$ was 69 months (or 5 years and 9 months). Roughly half of mothers were employed at wave 1, 68\% of mothers were married and $52 \%$ of mothers participated in WIC during their pregnancy. Finally, the majority of families had established rules concerning food (75\%) and ate dinner together most days of the week (mean $=5 \cdot 97$ ).

Next, we explored whether breast-feeding duration independently influences junk food consumption and whether this influence varies by level of SES. The results of these analyses are displayed in Table 2 . Model 1 of Table 2 revealed that a longer duration of breast-feeding was indeed associated with significant reductions in offspring 
junk food consumption, net of covariates $(P<0 \cdot 01)$. The standardized effect associated with breast-feeding duration $(\beta=-0 \cdot 07, P<0 \cdot 01)$, however, was roughly half the size of the standardized effect of SES $(\beta=-0 \cdot 15, P<0 \cdot 01)$, yet was similar in size to the standardized effect of food rules $(\beta=-0 \cdot 08, P<0 \cdot 01)$. The results of Model 1 also indicated that male children exhibited higher levels of junk

Table 1 Descriptive statistics of the nationally representative sample of US-born children, Early Childhood Longitudinal Study: Birth Cohort (ECLS-B), 2001-2007/2008

\begin{tabular}{lrcc}
\hline Variable & Mean & \multicolumn{1}{c}{ SD } & Range \\
\hline 1. Junk food consumption & 1.58 & 0.81 & $0-5.5$ \\
2. Fast-food consumption & 0.91 & 0.84 & $0-6$ \\
3. Soda consumption & 1.50 & 1.50 & $0-6$ \\
4. Salty snack consumption & 1.71 & 1.24 & $0-6$ \\
5. Sweets consumption & 2.22 & 1.32 & $0-6$ \\
6. Breast-feeding duration & 4.34 & 5.61 & $0-21$ \\
7. SES & 0.00 & 1.00 & -2.41 to 2.59 \\
8. White & 0.45 & 0.50 & $0-1$ \\
9. Hispanic & 0.17 & 0.38 & $0-1$ \\
10. Black & 0.16 & 0.36 & $0-1$ \\
11. Child sex (male =1) & 0.51 & 0.50 & $0-1$ \\
12. Child age (in months) & 68.65 & 4.72 & $57-86$ \\
13. Child attachment security & 0.60 & 0.49 & $0-1$ \\
14. Maternal age at birth & 27.49 & 6.36 & $15-50$ \\
15. Marital status (married $=1$ ) & 0.68 & 0.47 & $0-1$ \\
16. Employment (employed $=1$ ) & 0.51 & 0.49 & $0-1$ \\
17. WIC participation & 0.52 & 0.50 & $0-1$ \\
18. Postpartum depression & 1.42 & 0.72 & $1-4$ \\
19. Maternal involvement & 3.02 & 0.62 & $1-4$ \\
20. Food rules & 0.75 & 0.43 & $0-1$ \\
21. Family meals & 5.97 & 1.80 & $0-7$ \\
\hline
\end{tabular}

SES, socio-economic status; WIC, Special Supplemental Nutrition Program for Women, Infants and Children. food consumption and that, relative to the reference category (i.e. other race/ethnicity), children of black and white mothers were more likely to eat junk food more often. Model 2 of Table 2 expands upon the findings of Model 1 by examining the interaction between breastfeeding duration and SES as a predictor of junk food consumption. The interaction emerged as significant and positive $(\beta=0.05, P<0.01)$, suggesting that the negative effect of breast-feeding duration on offspring junk food consumption was diminished (i.e. was more positive) among higher-SES individuals and was enhanced (i.e. was more negative) among lower-SES individuals.

The relationship between breast-feeding duration and offspring junk food consumption was explored further by dividing the sample into subgroups distinguished by race/ ethnicity. The results of these analyses appear in Models 1 , 3 and 5 of Table 3. The findings indicated that breastfeeding duration significantly reduced offspring junk food consumption among whites and blacks, but not among Hispanics. The $z$ tests for the equality of regression coefficients revealed that breast-feeding had a significantly stronger negative effect on junk food consumption among whites than among Hispanics $(z=-2 \cdot 00)$. Breast-feeding coefficients, however, were not significantly different between blacks and whites $(z=-0.73)$ or blacks and Hispanics $(z=-1.56)$. In addition, Models 1,3 and 5 revealed that SES was significantly and negatively associated with junk food consumption across all racial/ethnic groups. The $z$ tests for the equality of regression coefficients, moreover, revealed that the negative association between SES and junk food consumption was significantly

Table 2 Breast-feeding duration and offspring junk food consumption, and moderation by socio-economic status (SES), in a nationally representative sample of US-born children, Early Childhood Longitudinal Study: Birth Cohort (ECLS-B), 2001-2007/2008

\begin{tabular}{|c|c|c|c|c|c|c|}
\hline \multirow[b]{3}{*}{ Variable } & \multicolumn{6}{|c|}{ Junk food consumption } \\
\hline & \multicolumn{3}{|c|}{ Model 1} & \multicolumn{3}{|c|}{ Model 2} \\
\hline & $b$ & $\beta$ & SE & $b$ & $\beta$ & $\mathrm{SE}$ \\
\hline Breast-feeding duration & $-0.05^{\star \star}$ & -0.07 & 0.01 & $-0.06^{\star *}$ & -0.08 & 0.01 \\
\hline SES & $-0 \cdot 12^{\star \star}$ & -0.15 & 0.02 & $-0 \cdot 12^{\star \star}$ & -0.15 & 0.02 \\
\hline White & $0.13^{\star \star}$ & 0.08 & 0.03 & $0.13^{\star \star}$ & 0.08 & 0.03 \\
\hline Hispanic & -0.02 & -0.01 & 0.04 & -0.01 & -0.01 & 0.04 \\
\hline Black & $0 \cdot 17^{\star \star}$ & 0.08 & 0.04 & $0.17^{\star *}$ & 0.08 & 0.04 \\
\hline Child sex $($ male $=1)$ & $0.04^{\star}$ & 0.03 & 0.02 & $0.04^{*}$ & 0.03 & 0.02 \\
\hline Child age (in months) & -0.01 & -0.02 & 0.01 & -0.01 & -0.02 & 0.01 \\
\hline Child attachment security & -0.03 & -0.02 & 0.02 & -0.03 & -0.02 & 0.02 \\
\hline Maternal age at birth & 0.00 & 0.00 & 0.00 & 0.00 & 0.00 & 0.00 \\
\hline Marital status (married $=1$ ) & -0.02 & -0.01 & 0.03 & -0.01 & -0.01 & 0.03 \\
\hline Employment (employed = 1) & -0.02 & -0.01 & 0.01 & -0.02 & -0.01 & 0.02 \\
\hline WIC participation & 0.02 & 0.01 & 0.03 & 0.02 & 0.01 & 0.03 \\
\hline Postpartum depression & 0.01 & 0.01 & 0.01 & 0.01 & 0.01 & 0.01 \\
\hline Maternal involvement & -0.01 & -0.01 & 0.02 & -0.01 & -0.01 & 0.02 \\
\hline Food rules & $-0 \cdot 15^{\star \star}$ & -0.08 & 0.03 & $-0 \cdot 15^{\star \star}$ & -0.08 & 0.03 \\
\hline Family meals & -0.01 & -0.01 & 0.01 & -0.01 & -0.02 & 0.01 \\
\hline Breast-feeding $\times$ SES & - & - & - & $0.04^{\star \star}$ & 0.05 & 0.01 \\
\hline$n$ & & 5732 & & & 5732 & \\
\hline
\end{tabular}

WIC, Special Supplemental Nutrition Program for Women, Infants and Children.

${ }^{\star} P<0.05,{ }^{\star *} P<0.01$. 


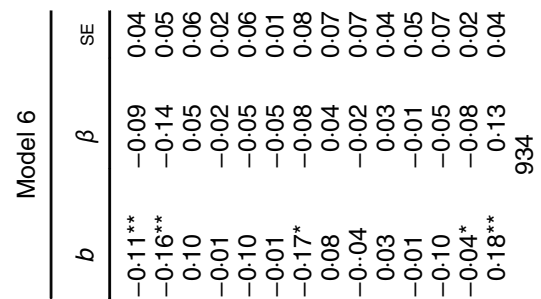

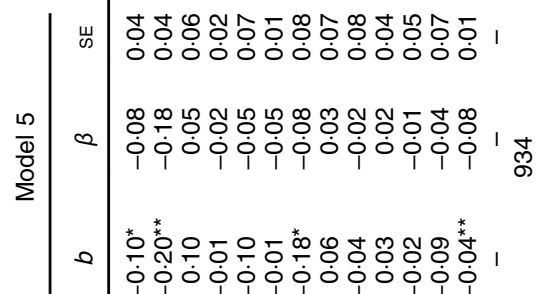

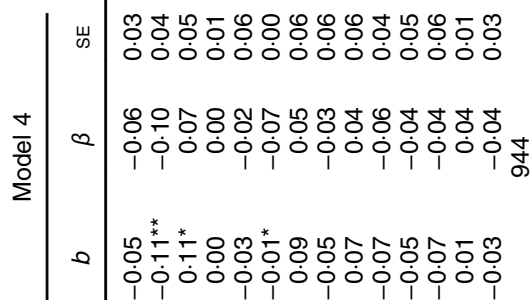

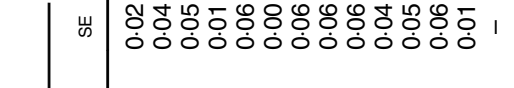

|

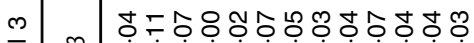

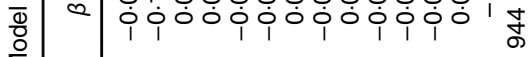

온

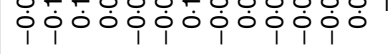

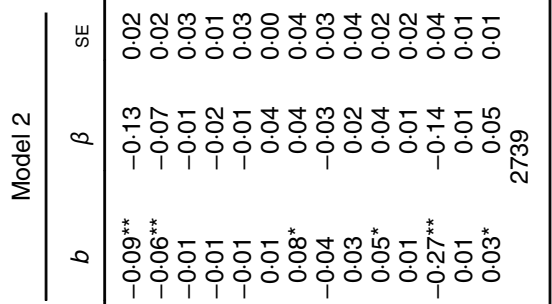

|

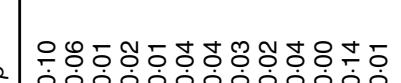

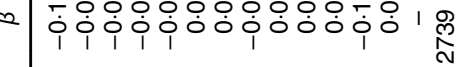

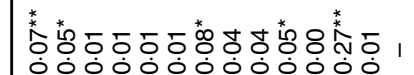

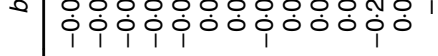

larger among blacks than among whites $(z=-3.66)$. SES coefficients, however, were not significantly different between blacks and Hispanics $(z=-1.40)$ or Hispanics and whites $(z=-1 \cdot 71)$.

After examining the influence of breast-feeding and SES across racial/ethnic groups, we proceeded to model the breast-feeding $\times$ SES interaction by racial/ethnic group to explore the possible role of three-way interactions between these variables in the prediction of offspring junk food consumption. These results are presented in Models 2, 4 and 6 of Table 3. A significant three-way interaction emerged between race/ethnicity, breast-feeding duration and SES. Specifically, the $z$ tests for the equality of regression coefficients revealed that breast-feeding duration was associated with significantly lower levels of offspring junk food consumption, but that this effect was especially pronounced among low-SES blacks (blacks $v$. whites: $z=-3.75, P<0.01$; blacks v. Hispanics: $z=-4 \cdot 20$; $P<0 \cdot 01$ ).

This significant three-way interaction is depicted in Fig. 1, which illustrates that, across racial/ethnic groups, breast-feeding duration had little to no effect on offspring junk food consumption among high-SES individuals. However, among low-SES individuals, the influence of breast-feeding duration on junk food consumption varied by race/ethnicity. In particular, breast-feeding duration had little association with junk food consumption among low-SES Hispanics, a significant negative association with junk food consumption among low-SES whites and a significantly larger negative association with junk food consumption among low-SES blacks. To illustrate, the predicted junk food consumption value among low-SES blacks who were never breast-fed was 1.84 , which equates to the 73rd percentile on the junk food consumption measure. Comparatively, the predicted junk food consumption value among low-SES blacks who attained the highest score on the breast-feeding measure was 0.79 , which equates to the 21st percentile on the junk food consumption measure (a difference of 52 percentile points).*

Finally, we conducted a series of ancillary analyses to examine whether the protective effect of breast-feeding duration on junk food consumption among low-SES blacks persisted across each of the junk food domains (i.e. fast foods, soda, salty snacks and sweets). The results are presented in Table 4 . The findings revealed that the breastfeeding $\times$ SES interaction was significantly and positively associated with all of the junk food domains among blacks, suggesting that the findings among the black sample are robust to junk food type $\dagger$ Figure 2 depicts the

* Comparatively, the percentile-point reduction in junk food consumption among low-SES whites was 26 (from the 66th percentile to the 40th percentile). Among low-SES Hispanics, it was 4 (from the 63rd percentile to the 59th percentile)

$\dagger$ Comparatively, none of the interactions were significantly predictive of any of the junk food domains among Hispanics. Only soda consumption was significantly predicted by the breast-feeding $\times$ SES interaction among whites. 


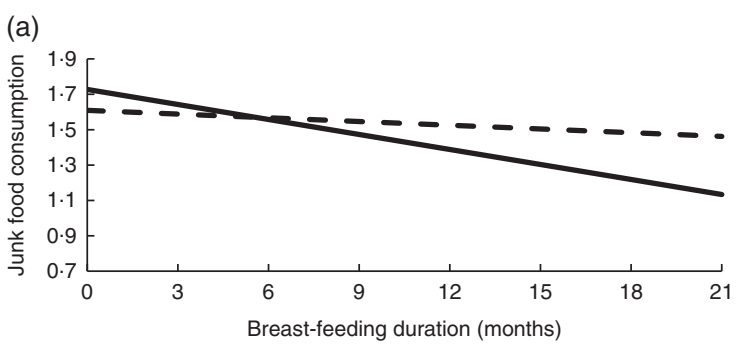

(b)

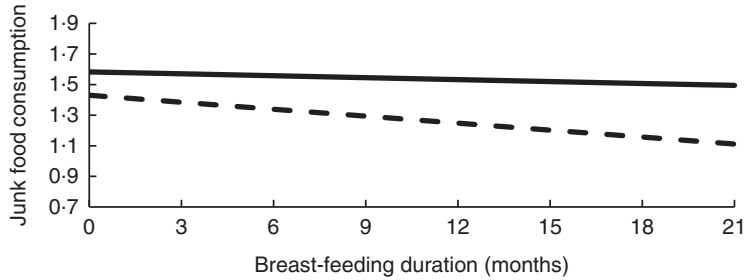

(c)

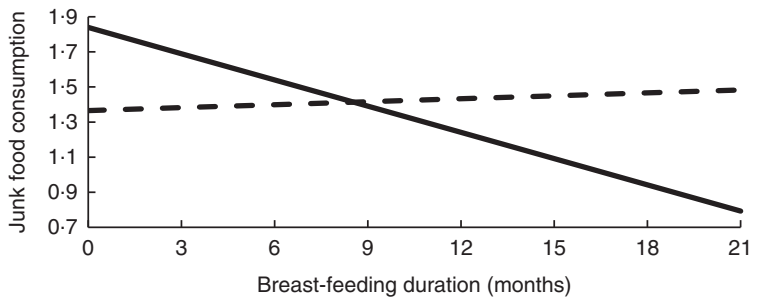

Fig. 1 Interaction between breast-feeding and socio-economic status (SES; — consumption by race/ethnicity (a, whites; b, Hispanics; c, blacks) among a nationally representative sample of US-born children, Early Childhood Longitudinal Study: Birth Cohort (ECLS-B), 2001-2007/2008. Low SES = below the median SES value; high SES $=$ above the median SES value

findings presented in Table 4. As illustrated in Fig. 2, a longer duration of breast-feeding among high-SES blacks had no consistent effect on junk food consumption across the four junk food domains. Conversely, a longer duration of breast-feeding was consistently associated with lower levels of fast-food, soda, salty snack and sweets consumption among low-SES blacks.

\section{Discussion}

Breast-feeding has been found to promote offspring health in myriad ways ${ }^{(1-6)}$. The present study adds to this body of research by revealing a negative relationship between breast-feeding and offspring junk food consumption, particularly among low-SES blacks. To be precise, a longer duration of breast-feeding was associated with lower levels of junk food consumption among low-SES blacks across the four measured domains: fast-food, soda, salty snack and sweets consumption. Associations between breast-feeding and each of the junk food dimensions, however, were not consistently detected among Hispanics

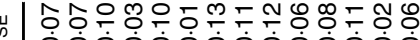

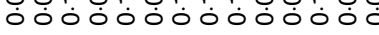

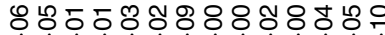

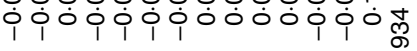

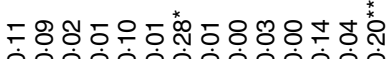

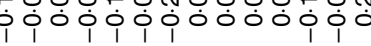

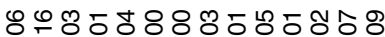

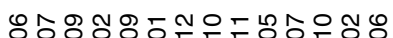

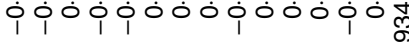
$\stackrel{0}{\Sigma}$

कृ
क

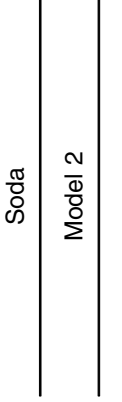

ш

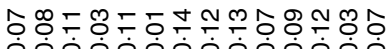

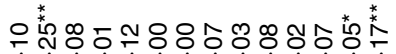

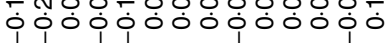

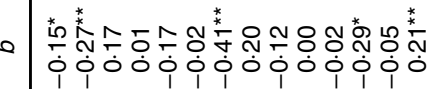

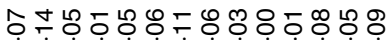

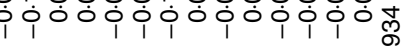

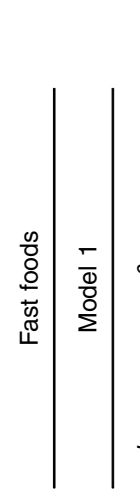

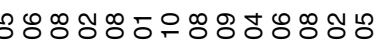

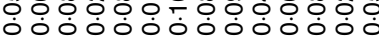

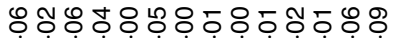
ílíl

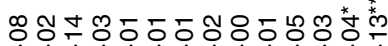

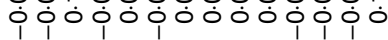

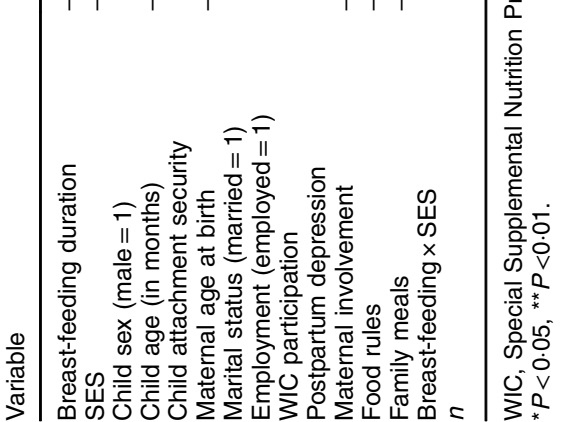



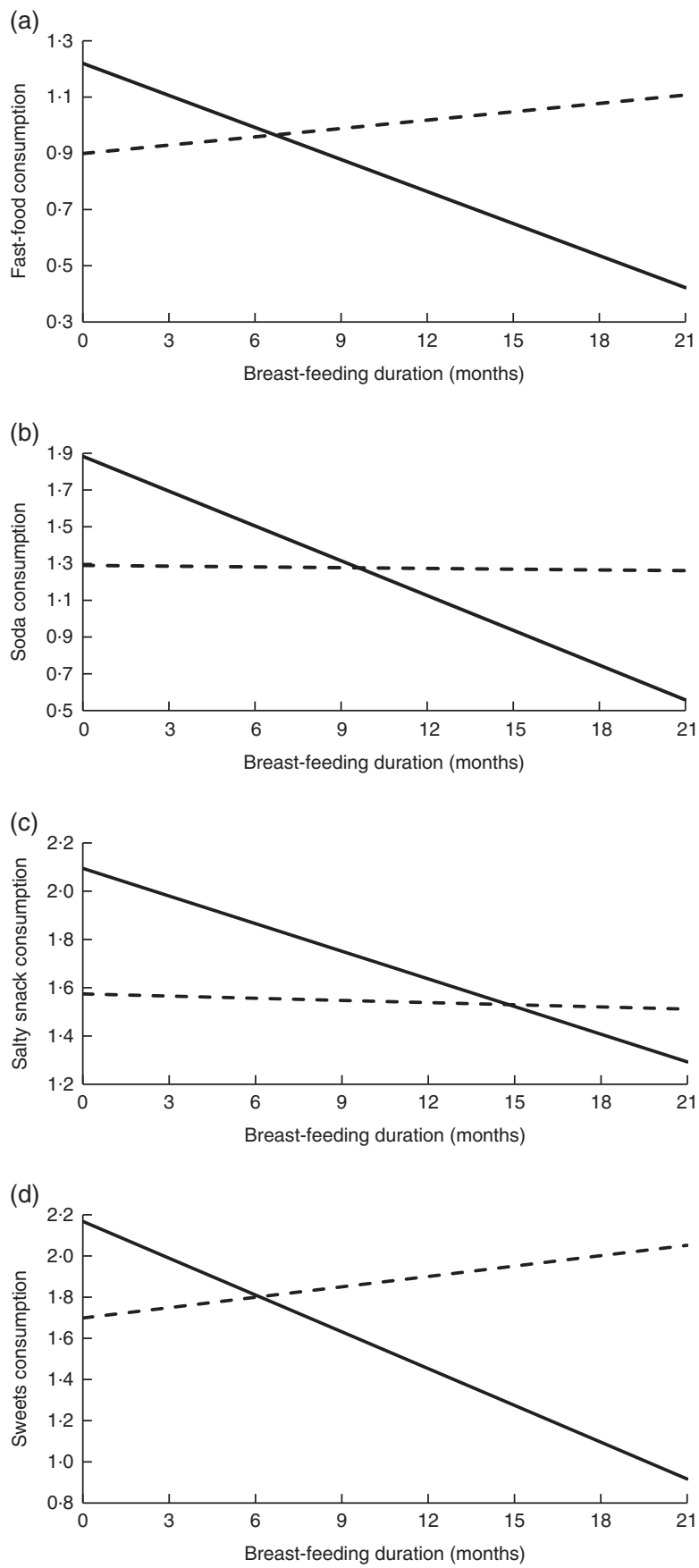

Fig. 2 Interaction between breast-feeding and socio-economic status (SES; — low SES; - - - , high SES) on junk food consumption (a, fast foods; b, soda; c, salty snacks; $d$, sweets) among blacks in a nationally representative sample of US-born children, Early Childhood Longitudinal Study: Birth Cohort (ECLS-B), 2001-2007/2008. Low SES=below the median SES value; high $S E S=$ above the median SES value

or whites, regardless of SES, nor were they detected for high-SES blacks, yielding a three-way interaction between breast-feeding, SES and race/ethnicity in the prediction of junk food consumption.

In light of the findings of the current study, it would be worthwhile to identify and promote strategies that assist women in their efforts to breast-feed. Our findings suggest that this may be particularly true in the case of low-SES black women, as breast-feeding among these women appears to have the greatest potential to improve offspring diet. To date, a number of effective breast-feeding promotion strategies for black women have been identified and/or are in development. Studies that evaluate social support interventions have found that a combination of strategies such as daily in-hospital visits, postpartum visits, prenatal visits or telephone calls from peer counsellors during the postpartum period, the provision of breast pumps, prenatal/childbirth classes taught by a nurse/peer counsellor, telephone support from either a nurse or a lactation consultant, and/or unlimited access to a nurse via pager have had a positive impact on breast-feed initiation and duration ${ }^{(21,47-55)}$. Social support that targets family members and the broader community has also significantly improved breast-feeding rates among black women. In a breast-feeding class for male partners, for instance, men learn the benefits of breast-feeding, ways to support their partner's decision to breast-feed and strategies to deal with those who may not be supportive of breast-feeding. Community Breastfeeding Gatherings, sponsored monthly by the African American Breastfeeding Network in Wisconsin, also promote breast-feeding by bringing together pregnant and breast-feeding mothers, their families and board-certified lactation consultants. Community Breastfeeding Gatherings begin with a familystyle meal and facilitate an informal and educational community dialogue, with recent events including a Peer Father Advocate component ${ }^{(56)}$.

Although it is important to identify breast-feeding promotion strategies that have been shown to be effective among black women, the present findings highlight the particular relevance of breast-feeding for the early health behaviours of children of a narrower subset of women: low-SES black women. A potentially useful point of intervention for these women is the WIC programme, as low-SES black women are, and have historically been, over-represented among WIC participants ${ }^{(57)}$. Any effort to promote breast-feeding among low-SES black women would be remiss if it did not consider the WIC programme as a unique opportunity to provide breast-feeding education and support to women who might not breast-feed otherwise. Not only has research revealed breast-feeding to be less common among WIC participants ${ }^{(58-64)}$, it has also revealed that black WIC participants have the lowest breast-feeding rates ${ }^{(59,63)}$ and are significantly less likely to receive breast-feeding advice from their health-care providers and/or WIC counsellors than white participants ${ }^{(59,64,65)}$.

In an effort to improve breast-feeding rates among WIC participants, especially black participants, the WIC programme expanded its breast-feeding education to include the Breastfeeding Peer Counseling Program ${ }^{(66,67)}$. The WIC peer counsellor must have breast-fed at least one 
child for 6 months or longer and be a current or previous WIC participant. Breastfeeding Peer Counseling Programs are offered as a means of providing educational, emotional and social support for new breast-feeding mothers ${ }^{(59,64)}$. Recent research has revealed that Breastfeeding Peer Counseling Programs have had positive effects on increasing breast-feeding initiation and continuation for black mothers enrolled in $\mathrm{WIC}^{(68,69)}$. Breastfeeding Peer Counseling Programs have discovered that black women tend to place high value on breast pumps as a matter of convenience and a way to exert control over breast-feeding ${ }^{(67)}$. Moreover, the educational component of the Breastfeeding Peer Counseling Program regarding the benefits of breast milk for infants has resulted in increasing breast-feeding rates among black WIC participants ${ }^{(59,64)}$. Due to many complex factors that affect lower-income black women's decision to breastfeed, a comprehensive approach should be implemented in order to improve breast-feeding initiation and duration rates among this subset of the population ${ }^{(58,64)}$.

The current study is the first to examine the association between breast-feeding and offspring junk food consumption separately for racial and SES groups using a nationally representative sample of US families. It is not, however, without its limitations. First, we would have preferred additional breast-feeding details (e.g. extracted milk $v$. feeding at the breast), including additional WHO core indicators of infant nutritional patterns (e.g. the ratio of breast milk $v$. formula, or predominant breast-feeding) but these details were not available in the data ${ }^{(70)}$.* $^{*}$ Second, we were only able to examine certain components of food consumption at one point in time (i.e. the kindergarten school year). Future research would benefit from an examination of a broader array of foods and changes in consumption over time. Third, due to data limitations, we were unable to fully examine maternal attitudes and education concerning general health and nutrition, despite accounting for food rules and routines within the familial context. Future studies should build upon our findings by exploring associations between breast-feeding duration, health awareness and nutritional knowledge among low-SES black women. Finally, apart from familial rules and routines regarding food consumption, the ECLS-B does not provide specific details concerning the dietary patterns of the parents. Maternal diet has been shown to influence offspring $\operatorname{diet}^{(71)}$; even so, prior studies linking breast-feeding to offspring diet are supportive even when they account for maternal $\operatorname{diet}^{(9)}$. As has been the case in prior research ${ }^{(10)}$, ancillary analyses of a sub-sample of participants that account for pre-pregnancy BMI resulted in no changes to our findings.

* Due to our emphasis on race/ethnicity and SES differences in the current study, it was also not feasible to examine the exclusivity of breast-feeding. There was not enough variation in the month-to-month measure for lowSES black women because exclusivity was infrequent among this population.
Despite the shortcomings of the current study, it represents an important extension of prior research as it considers ways that dietary patterns during childhood can be differentially shaped by breast-feeding practices across racial and socio-economic subsets of the population. Although we found the association between breast-feeding and child diet to be more pronounced among low-SES black women and children, it is important to promote breast-feeding for all racial/ethnic and SES groups due to the many health benefits associated with this practice $^{(1-6)}$. Efforts to promote breast-feeding among women require a comprehensive approach that seeks to shape health attitudes, enhance nutrition education and buttress social support systems. This comprehensive approach to developing interventions that facilitate breast-feeding may be particularly important for low-SES black women. Future research should aim to replicate and build upon the present study by (i) uncovering mechanisms that might link breastfeeding duration to offspring diet and (ii) exploring exactly how these processes might vary across racial and SES groups. Doing so can help to elucidate strategies that promote breast-feeding among low-SES black women and improve nutritional habits in low-SES black children, setting the stage for future health and well-being.

\section{Acknowledgements}

Acknowledgements: The authors would like to acknowledge the Institute of Education Sciences and its sponsoring agencies, which provided the corresponding author with access to the ECLS-B data. Financial support: This research received no specific grant from any funding agency in the public, commercial or not-for-profit sectors. Conflict of interest: The authors declare that they have no conflicts of interest. Authorship: D.B.J. conceived of the study, developed the research design, analysed the data, tabulated the results and drafted the manuscript. K.R.J. drafted the manuscript and aided in the interpretation of the findings. Both authors reviewed and approved the final version of the manuscript. Ethics of human subject participation: This study was conducted according to the guidelines laid down in the Declaration of Helsinki and all procedures involving human subjects were approved by the University of Texas at San Antonio. Written informed consent was obtained from all participants.

\section{References}

1. Hanson LA (1998) Breastfeeding provides passive and likely long-lasting active immunity. Ann Allergy Asthma Immunol 81, 523-537.

2. Duijts L, Ramadhani MK \& Moll HA (2009) Breastfeeding protects against infectious diseases during infancy in industrialized countries. A systematic review. Matern Child Nutr 5, 199-210. 
3. Martin RM, Gunnell D \& Smith GD (2005) Breastfeeding in infancy and blood pressure in later life: systematic review and meta-analysis. Am J Epidemiol 161, 15-26.

4. Cushing AH, Samet JM, Lambert WE et al. (1998) Breastfeeding reduces risk of respiratory illness in infants. $A m \mathrm{~J}$ Epidemiol 147, 863-870.

5. Harder T, Bergmann R, Kallischnigg G et al. (2005) Duration of breastfeeding and risk of overweight: a meta-analysis. Am J Epidemiol 162, 397-403.

6. Yan J, Liu L, Zhu Y et al. (2014) The association between breastfeeding and childhood obesity: a meta-analysis. BMC Public Health 14, 1267.

7. Abraham EC, Godwin J, Sherriff A et al. (2012) Infant feeding in relation to eating patterns in the second year of life and weight status in the fourth year. Public Health Nutr 15, 1705-1714.

8. Husk JS \& Keim SA (2016) Breastfeeding and dietary variety among preterm children aged 1-3 years. Appetite 99, 130-137.

9. Jones L, Moschonis G, Oliveira A et al. (2015) The influence of early feeding practices on healthy diet variety score among pre-school children in four European birth cohorts. Public Health Nutr 18, 1774-1784.

10. Perrine CG, Thompson FE \& Scanlon K (2014) Breastfeeding duration is associated with child diet at 6 years. Pediatrics 134, Suppl. 1, S50-S55.

11. Scott JA, Chih TY \& Oddy WH (2012) Food variety at 2 years of age is related to duration of breastfeeding. Nutrients $\mathbf{4}$, 1464-1474.

12. Soldateli B, Vigo A \& Giugliani ERJ (2016) Effect of pattern and duration of breastfeeding on the consumption of fruits and vegetables among preschool children. PLoS One $\mathbf{1 1}$ e0148357.

13. Leech RM, McNaughton SA \& Timperio A (2015) Clustering of diet, physical activity and sedentary behaviour among Australian children: cross-sectional and longitudinal associations with overweight and obesity. Int J Obes (Lond) 39, 1079-1085.

14. Swinburn BA, Caterson I, Seidell JC et al. (2004) Diet, nutrition and the prevention of excess weight gain and obesity. Public Health Nutr 7, 123-146.

15. Taveras EM, Scanlon KS, Birch L et al. (2004) Association of breastfeeding with maternal control of infant feeding at age 1 year. Pediatrics 114, e577-e583.

16. Forste R, Weiss J \& Lippincott E (2001) The decision to breastfeed in the United States: does race matter? Pediatrics 108, 291-296.

17. Mickens AD, Modeste N, Montgomery S et al. (2009) Peer support and breastfeeding intentions among black WIC participants. J Hum Lact 25, 157-162.

18. Forste R \& Hoffmann JP (2008) Are US mothers meeting the Healthy People 2010 breastfeeding targets for initiation, duration, and exclusivity? The 2003 and 2004 National Immunization Surveys. J Hum Lact 24, 278-288.

19. Gibbs BG \& Forste R (2014) Socioeconomic status, infant feeding practices and early childhood obesity. Pediatr Obes 9, 135-146.

20. Heck KE, Braveman P, Cubbin C et al. (2006) Socioeconomic status and breastfeeding initiation among California mothers. Public Health Rep 121, 51-59.

21. Chapman DJ \& Pérez-Escamilla R (2012) Breastfeeding among minority women: moving from risk factors to interventions. Adv Nutr 3, 95-104.

22. Kaufman L, Deenadayalan S \& Karpati A (2010) Breastfeeding ambivalence among low-income African American and Puerto Rican women in north and central Brooklyn. Matern Child Health J 14, 696-704.

23. Celi AC, Rich-Edwards JW, Richardson MK et al. (2005) Immigration, race/ethnicity, and social and economic factors as predictors of breastfeeding initiation. Arch Pediatr Adolesc Med 159, 255-260.
24. Scott JA, Landers MCG, Hughes RM et al. (2001) Factors associated with breastfeeding at discharge and duration of breastfeeding. J Paediatr Child Health 37, 254-261.

25. Fuemmeler BF, Stroo M, Lee CT et al. (2015) Racial differences in obesity-related risk factors between 2-year-old children born of overweight mothers. J Pediatr Psychol 40, 649-656.

26. Garnett BR, Rosenberg KD \& Morris DS (2013) Consumption of soda and other sugar-sweetened beverages by 2-year-olds: findings from a population-based survey. Public Health Nutr 16, 1760-1767.

27. Guerrero AD, Mao C, Fuller B et al. (2016) Racial and ethnic disparities in early childhood obesity: growth trajectories in body mass index. J Racial Ethn Health Disparities 3, 129-137.

28. Armstrong J \& Reilly JJ (2002) Breastfeeding and lowering the risk of childhood obesity. Lancet 359, 2003-2004.

29. Burdette HL \& Whitaker RC (2007) Differences by race and ethnicity in the relationship between breastfeeding and obesity in preschool children. Ethn Dis 17, 467.

30. Butte NF (2009) Impact of infant feeding practices on childhood obesity. J Nutr 139, issue 2, 412S-416S.

31. Ehrenthal DB, Wu P \& Trabulsi J (2016) Differences in the protective effect of exclusive breastfeeding on child overweight and obesity by mother's race. Matern Child Health J 20, 1971-1979.

32. Grummer-Strawn LM \& Mei Z (2004) Does breastfeeding protect against pediatric overweight? Analysis of longitudinal data from the Centers for Disease Control and Prevention Pediatric Nutrition Surveillance System. Pediatrics 113, e81-e86.

33. Evenhouse E \& Reilly S (2005) Improved estimates of the benefits of breastfeeding using sibling comparisons to reduce selection bias. Health Serv Res 40, 1781-1802.

34. Hayatbakhsh MR, O'Callaghan MJ, Bor W et al. (2012) Association of breastfeeding and adolescents' psychopathology: a large prospective study. Breastfeed Med 7, 480-486.

35. Heikkilä KA, Sacker Y, Kelly MJ et al. (2011) Breastfeeding and child behaviour in the Millennium Cohort Study. Arch Dis Child 96, 635-642.

36. Reynolds D, Hennessy E \& Polek E (2014) Is breastfeeding in infancy predictive of child mental well-being and protective against obesity at 9 years of age? Child Care Health Dev 40, 882-890.

37. Mollborn S \& Lovegrove PJ (2011) How teenage fathers matter for children: evidence from the ECLS-B.J Fam Issues 32, 3-30.

38. Tucker-Drob EM, Rhemtulla M, Harden KP et al. (2011) Emergence of a gene $\times$ socioeconomic status interaction on infant mental ability between 10 months and 2 years. Psychol Sci 22, 125-133.

39. Jaccard J, Wan CK \& Turrisi R (1990) The detection and interpretation of interaction effects between continuous variables in multiple regression. Multivar Behav Res 25, 467-478.

40. Jackson DB (2016) The association between breastfeeding duration and attachment: a genetically informed analysis. Breastfeed Med 11, 297-304.

41. Bost KK, Wiley AR, Fiese B et al. (2014) Associations between adult attachment style, emotion regulation, and preschool children's food consumption. J Dev Behav Pediatr 35, 50-61.

42. Vogel A, Hutchison BL \& Mitchell EA (1999) Factors associated with the duration of breastfeeding. Acta Paediatr 88, 1320-1326.

43. Northstone K \& Emmett P (2005) Multivariate analysis of diet in children at four and seven years of age and associations with socio-demographic characteristics. Eur J Clin Nutr 59, 751-760. 
44. Scott JA, Binns CW, Oddy WH et al. (2006) Predictors of breastfeeding duration: evidence from a cohort study. Pediatrics 117, e646-e655.

45. Ryan AS \& Zhou W (2006) Lower breastfeeding rates persist among the Special Supplemental Nutrition Program for Women, Infants, and Children participants, 1978-2003. Pediatrics 117, 1136-1146.

46. Paternoster R, Brame R, Mazerolle P et al. (1998) Using the correct statistical test for the equality of regression coefficients. Criminology 36, 859-866.

47. Anderson AK, Damio G, Young S et al. (2005) A randomized trial assessing the efficacy of peer counseling on exclusive breastfeeding in a predominantly Latina low-income community. Arch Pediatr Adolesc Med 159, 836-841.

48. Bonuck KA, Trombley M, Freeman K et al. (2005) Randomized, controlled trial of a prenatal and postnatal lactation consultant intervention on duration and intensity of breastfeeding up to 12 months. Pediatrics 116, 1413-1426.

49. Bonuck KA, Freeman K \& Trombley M (2006) Randomized controlled trial of a prenatal and postnatal lactation consultant intervention on infant health care use. Arch Pediatr Adolesc Med 160, 953-960.

50. Bunik M, Shobe P, O'Connor ME et al. (2010) Are 2 weeks of daily breastfeeding support insufficient to overcome the influences of formula? Acad Pediatr 10, 21-28.

51. Chapman DJ, Morel K, Bermudez-Millan A et al. (2011) Breastfeeding education and support trial for obese women: effects of a specialized peer counseling intervention on breastfeeding and health outcomes. J Hum Lact 27, 75-76.

52. Ickovics JR, Kershaw TS, Westdahl C et al. (2007) Group prenatal care and perinatal outcomes: a randomized controlled trial. Obstet Gynecol 110, 330-339.

53. Petrova A, Ayers C, Stechna S et al. (2009) Effectiveness of exclusive breastfeeding promotion in low-income mothers: a randomized controlled study. Breastfeed Med 4, 63-69.

54. Pugh LC, Milligan RA, Frick KD et al. (2002) Breastfeeding duration, costs, and benefits of a support program for lowincome breastfeeding women. Birth 29, 95-100.

55. Wolfberg AJ, Michels KB, Shields W et al. (2004) Dads as breastfeeding advocates: results from a randomized controlled trial of an educational intervention. Am J Obstet Gynecol 191, 708-712.

56. Centers for Disease Control and Prevention (2014) Breastfeeding Report Card - United States, 2014. Atlanta, GA: Centers for Disease Control and Prevention; available at http://www. cdc.gov/breastfeeding/pdf/2014breastfeedingreportcard.pdf

57. Thorn B, Tadler C, Huret N et al. (2015) WIC Participant and Program Characteristics 2014. Prepared by Insight Policy Research under Contract No. AG-3198-C-11-0010. Alexandria, VA: US Department of Agriculture, Food and Nutrition Service.
58. Dunn RL, Kalich KA, Fedrizzi R et al. (2015) Barriers and contributors to breastfeeding in WIC mothers: a social ecological perspective. Breastfeed Med 10, 493-501.

59. Evans K, Labbok M \& Abrahams SW (2011) WIC and breastfeeding support services: does the mix of services offered vary with race and ethnicity? Breastfeed Med $\mathbf{6}$, 401-406.

60. Gregory EF, Gross SM, Nguyen TQ et al. (2016) WIC participation and breastfeeding at 3 months postpartum. Matern Child Health J 20, 1735-1744.

61. Hurley KM, Black MM, Papas MA et al. (2008) Variation in breastfeeding behaviours, perceptions, and experiences by race/ethnicity among a low-income statewide sample of Special Supplemental Nutrition Program for Women, Infants, and Children (WIC) participants in the United States. Matern Child Nutr 4, 95-105.

62. Jensen E (2012) Participation in the Supplemental Nutrition Program for Women, Infants and Children (WIC) and breastfeeding: national, regional, and state level analyses. Matern Child Health J 16, 624-631.

63. Marshall C, Gavin L, Bish C et al. (2013) WIC participation and breastfeeding among White and Black mothers: data from Mississippi. Matern Child Health J 17, 1784-1792.

64. Robinson K, VandeVusse L \& Foster J (2016) Reactions of low-income African American women to breastfeeding peer counselors. J Obstet Gynecol Neonatal Nurs 45, 62-70.

65. Beal AC, Kuhlthau K \& Perrin JM (2003) Breastfeeding advice given to African American and white women by physicians and WIC counselors. Public Health Rep 118, 368-376.

66. Chapman DJ, Morel K, Anderson AK et al. (2010) Review: breastfeeding peer counseling: from efficacy through scale-up. J Hum Lact 26, 314-326.

67. Gross TT, Powell R, Anderson AK et al. (2015) WIC peer counselors' perceptions of breastfeeding in African American women with lower incomes. J Hum Lact 31, 99-110.

68. Baumgartel KL, Spatz DL \& American Academy of Nursing Expert Breastfeeding Panel (2013) WIC (the Special Supplemental Nutrition Program for Women, Infants, and Children): policy versus practice regarding breastfeeding. Nurs Outlook 61, 466-470.

69. Campbell LA, Wan J, Speck PM et al. (2014) Women, Infant and Children (WIC) peer counselor contact with first time breastfeeding mothers. Public Health Nurs 31, 3-9.

70. World Health Organization (2010) Indicators for Assessing Infant and Young Child Feeding Practices. Part II: Measurement. Geneva: WHO.

71. Durão C, Severo M, Oliveira A et al. (2016) Association of maternal characteristics and behaviours with 4-year-old children's dietary patterns. Matern Child Nutr (Epublication ahead of print version). 